\title{
Non-homogeneous polymer model for wave propagation and its rheological response
}

\author{
Rajneesh Kakar* \\ Principal, DIPS Polytechnic College, Hoshiarpur-146001, INDIA \\ *Corresponding author: e-mail: rkakar_163@rediffmail.com, Tel. +919915716560, FAX. +911886237166
}

\begin{abstract}
This article concerns certain aspects of four parameter polymer models to study harmonic waves in the non-homogeneous polymer rods of varying density. There are two sections of this paper, in first section, the rheological behaviour of the model is discussed numerically and then it is solved analytically with the help of Friedlander Series using Eikonal equation of optics. The model is chosen for studying viscous, elastic and retarded elastic response to shearing stress. In another section, the applicability of the developed model is studied through wave propagation in polymer non-homogeneous rods. We have used linear partial differential equations for finding the dispersion equation of waves in the polymers. The rheological behaviour of four-parameter viscoelastic models under dynamic loading is also discussed in detail. All the cases taken in this study are discussed analytically and numerically with MATLAB.
\end{abstract}

Keywords: Harmonic Waves; Friedlander Series; Inhomogeneous; Varying Density.

\section{Introduction}

Many branches of science like seismology, earth-quake \& civil engineering, acoustics, optics, bio-mechanics, ocean engineering, electronics \& communication engineering, mechanical engineering, applied physics and applied mathematics are working experimentally and analytically to study the characteristics of engineering materials by wave propagation in homogeneous and non-homogeneous media for the last few decades. For the elastic and viscoelastic waves, a long list of references is available in the monographs of Victorov (1958), Milkowitz, J (1966) and Kolsky (1966).

In solid materials, during rest position, the volume elements retain their relative positions and orientations alike. On the other hand, when the solid is under the action of external forces such as elastic stresses and strains, the volume elements get departed from the original position. Therefore, the subject of elasticity of crystals has its own importance in several circumstances. In recent years, the elastic behaviour of polycrystalline solids, used as the materials in engineering construction, are of great practical importance.

Mathematical models to analysis of wave propagation in homogeneous materials have a centuries old history. Modeling problems actually is a study of mechanical properties of materials. The polymer models have specific characteristics which distinguish them from elastic models. The elastic materials store maximum of the energy (100\%) due to deformation but viscoelastic materials do not do this. The dissipation of energy in polymer materials is known as hysteresis. Nearly, all materials behave like some viscoelastic response. However, some common materials such as steel or quartz do not deviate much from linear elasticity at room temperature. But synthetic rubber, wood, and biological tissue and metals at high temperature show significant viscoelastic effects.

In the earlier times, sufficient interest has been led on wave propagation in that material whose mechanical characteristics and density are functions of space i.e. non-homogeneous engineering material. Early attempt in this field was made by Victorov (1958) established the fundamental mathematically model of the problem for isotropic material properties have been taken into various 
forms: exponential (Restovtsov \& Khranexskaia, 1973; Rao and Goda, 1978): integral power law (Mukhopacryar 1979; Chandra 1980; Narain 1980) and binominal (Singh and Dhaliwal, 1980; Wantanabe 1981); Singh Chopra \& Gogna, 1978) studied the SHwaves propagation in a laterally and vertically heterogeneous layered media where as Breuer and Shlomo (1970), Gaiduk (1971), Kriekaja (1971), Turoan and Mengi (1970), Rous (1978), Singh (1978) and Singh and Singh (1979) have treated the wave propagation problems in Non-homogeneous Viscoelastic media.

On the basis of the theory of elasticity, the propagation of harmonic waves in isotropic or anisotropic materials has been evaluated numerically by White (1981), Mirsky (1965) and Tsai (1991). To explain the soil behaviour, Murayama and Shibata (1961) and Schiffman et al. (1964) developed five and seven parameter models. Moodie (1973) presented research paper on propagation, reflection and transmission of transient cylindrical shear waves in non-homogeneous four-parameter viscoelastic media. Kakar et al. (2012-2013) and Kaur et al. (2012-2013) have studied four and five parameter viscoelastic models for wave propagation and dynamic loading.

But in this study, authors have considered the specimen is non-homogeneous i.e. density, rigidity and viscosity of the rod is space dependent. In this paper, the wave equation is approximated by using WKB theory. The displacements are assumed to be small under isothermal conditions, the linear constitutive laws hold. Time dependent displacement and stress boundary conditions are employed for calculating the relations for displacement and stress. The rods are assumed to be initially unstressed and at rest. In this study, it is assumed that density ' $\rho$ ', rigidity ' $G$ ' and viscosity ' $\eta$ ' of the specimen i.e. rod are space dependent and obey the harmonic laws as $\rho=\rho_{0} e^{2 i \alpha_{1} x}, k=k_{0} e^{2 i \alpha_{2} x}, \eta=\eta_{0} e^{2 i \alpha_{3} x}$. The various graphs are plotted to show the effect of nonhomogeneity on the velocity of waves.

\section{Formulation of Problem}

Let us consider wave is propagating in one dimensional non-homogeneous semi-infinite rod, the end of the rod is kept at $\mathrm{x}=0$. We consider the four parameter model with two springs $S_{1}\left(G_{1}\right), S_{2}\left(G_{2}\right)$ and two dash-pots $D_{1}\left(\eta_{1}\right), D_{2}\left(\eta_{2}\right)$ with viscoelasticity $\eta_{1}$ and $\eta_{2}$ respectively (Figure 1 ). The springs represent recoverable elastic response and dash pot represents elements in the structure giving rise to viscous drag. Here $G_{1}$ and $G_{2}$ are elastic parameters, $\eta_{1}$ and $\eta_{2}$ are viscoelastic parameters. Let $\sigma$ be the stress and $a$ be the strain in the model. Let $a_{1}$ be the strain in $S_{1}\left(G_{1}\right), a_{2}$ be the strain along dashpot $D_{1}\left(\eta_{1}\right)$ and $a_{3}$ be the strain in the Kelvin model. Figure 1 represents the sketch of the standard four parameter viscoelastic models. The stress v/s strain behavior for constant stress $(\sigma)$ with time $\left(t_{a}\right)$ has been shown in Figure 1 . Here, $G_{1}=\lambda_{1}+2 \mu_{1}, G_{2}=\lambda_{2}+2 \mu_{2}$ are the modulli of elasticity, $\eta_{1}, \eta_{2}$ are Newtonian viscosities coefficients and taken as functions of ' $x$ ' in the non-homogeneous case.
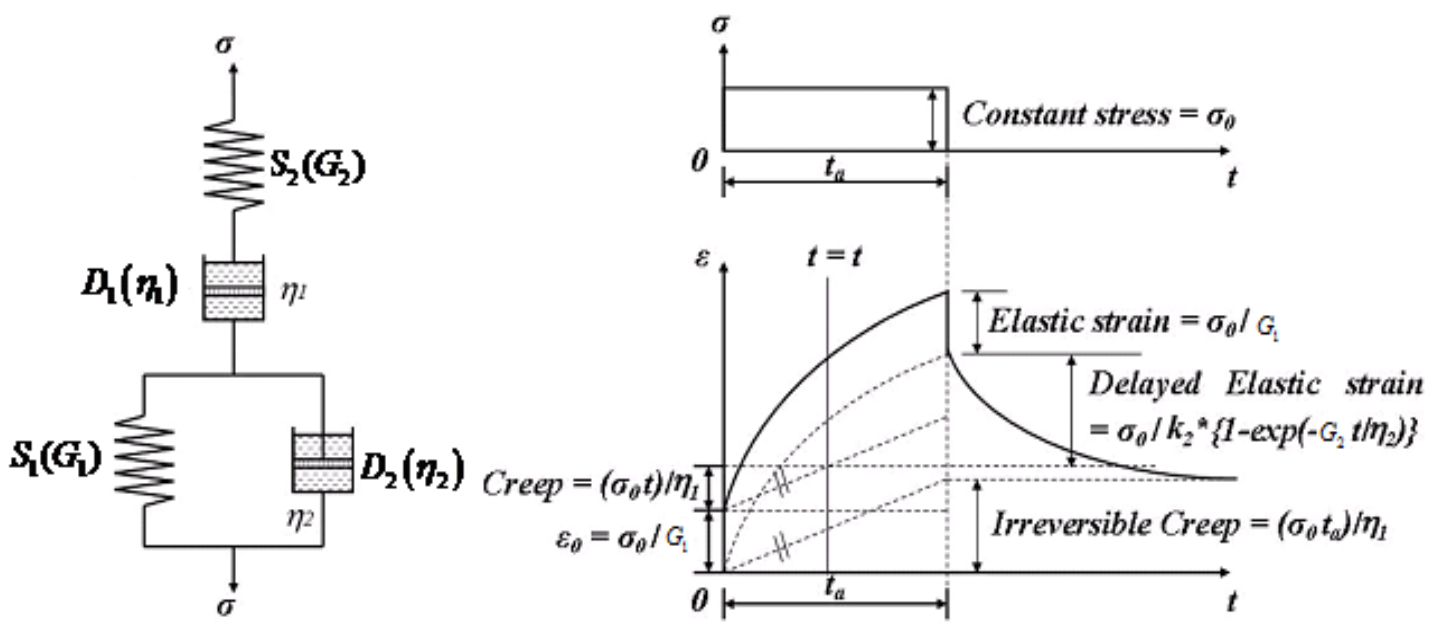

Figure 1. Rheological model and its response

The stress-strain relation for the four parameter viscoelastic models are constituted by the equations, Kakar et al. (2013)

$$
\sigma=G_{1} a_{1}, \sigma=\eta_{1} \dot{a}_{2}, \sigma=G_{2} a_{3}, \sigma=\eta_{2} \dot{a}_{3} .
$$


Eliminating $a_{1}, a_{2}, a_{3}$ from Eq. (1) we get the constitute equation for the four parameter model:

$$
\ddot{\sigma}+\left(\frac{G_{1}}{\eta_{2}}+\frac{G_{2}}{\eta_{2}}+\frac{G_{1}}{\eta_{1}}\right) \dot{\sigma}+\left(\frac{G_{1} G_{2}}{\eta_{1} \eta_{2}}\right) \sigma=G_{1} \ddot{a}+\frac{G_{1} G_{2}}{\eta_{2}} \dot{a}
$$

The stress strain equation for four parameter model is of general form:

$$
\ddot{\sigma}+B_{1} \dot{\sigma}+B_{0} \sigma=A_{2} \ddot{a}+A_{1} \dot{a}
$$

Where $B_{i}$ 's and $A_{i}$ 's are the coefficients made up of combinations of the $G_{1}, G_{2}$ and $\eta_{1}, \eta_{2}$ and depend upon the specific arrangement of the elements in the model. In operator form the Eq. (2) can be written as

$$
\left\{\partial_{t}^{2}+B_{1} \partial_{t}+B_{0}\right\} \sigma=\left\{A_{2} \partial_{t}^{2}+A_{1} \partial_{t}\right\} a
$$

The equation of motion and strain-displacement relation is given by

$$
\begin{gathered}
\frac{\partial \sigma}{\partial x}=\rho \frac{\partial^{2} U}{\partial t^{2}} \\
a=\frac{\partial U}{\partial x}
\end{gathered}
$$

Where $\rho=\rho(x)$ is the variable density of the material.

Differentiating Eq.(5) w.r.t $X$,we get

$$
-\frac{1}{\rho^{2}} \frac{\partial \rho}{\partial x} \frac{\partial \sigma}{\partial x}+\frac{1}{\rho} \frac{\partial^{2} \sigma}{\partial x^{2}}=u_{, x t t}
$$

Differentiating Eq.(6) w.r.t. $t$, we get

$$
\frac{\partial a}{\partial t}=\frac{\partial}{\partial t}\left(\frac{\partial u}{\partial x}\right)
$$

Again differentiating w.r.t. $t$,

$$
\frac{\partial^{2} a}{\partial t^{2}}=\frac{\partial}{\partial t}\left\{\frac{\partial}{\partial t}\left(\frac{\partial u}{\partial x}\right)\right\}=u_{, t t x}=u_{, x t t}
$$

Using Eq. (7) and Eq. (8), Eq. (3) gives

$$
\sigma_{, t t t}+, B_{1} \sigma_{, t t}+B_{0} \sigma_{, t}=\frac{1}{\rho}\left\{A_{2} \sigma_{, x x t}-A_{2}(\log \rho)_{, x} \sigma_{, x t}+A_{1} \sigma_{, x x}-A_{1}(\log \rho)_{, x} \sigma_{, x}\right\}
$$

\section{Method of Solution}

Let the solution $\sigma(x, t)$ of Eq. (9) may be represented by the series, Friedlander (1947)

$$
\sigma(x, t)=\sum_{n=0}^{\infty} A_{n}(x) F_{n}\{t-h(x)\}, \quad A_{0} \neq 0
$$

Where,

$$
\left.F_{n}^{\prime}=F_{n-1} \text { (where, } \mathrm{n}=1,2,3 \ldots \ldots \ldots \ldots\right) \text { with } F_{n, t}=F_{n-1} \text { and } F_{n, x}=-h_{, x} F_{n-1}
$$

and for $n<0$ assume that $A_{n}=0$ and the derivatives of $\sigma$ may be obtained by term-wise differentiation of Eq. (10), the prime in Eq. (11) denotes differentiation with respect to the argument concerned, and by using Eq. (10) and Eq.(11) we relate all $F_{n}^{\prime}$ s to $F_{0}$ by successive integrations.

The Solution of equation Eq. (9) in the form of Eq. (10) can be obtained by taking a phase function $h(x), h(x)$ satisfies the Eikonal equation of geometrical optics, Moodie (1973) 


$$
\left(\frac{d h(x)}{d x}\right)^{2}=\frac{\rho}{G_{1}}=\frac{1}{c^{2}}
$$

Where $\mathrm{c}=\mathrm{c}(\mathrm{x})$ is the variable wave speed for elastic longitudinal waves in a medium whose modulus of elasticity $G_{1}$.Using, Eq.(10), Eq.(11) and the successive derivatives of $\sigma(x, t)$ w.r.t. ' $t$ ' and ' $x$ ' in equation Eq.(9), we get

$$
\begin{gathered}
A_{n} F_{n-3}+\left(\frac{G_{1}}{\eta_{2}}+\frac{G_{2}}{\eta_{2}}+\frac{G_{1}}{\eta_{1}}\right) A_{n} F_{n-2}+\left(\frac{G_{1} G_{2}}{\eta_{1} \eta_{2}}\right) A_{n} F_{n-1} \\
=\frac{G_{1}}{\rho}\left\{A_{n}^{\prime \prime} F_{n-1}-2 A_{n}^{\prime} F_{n-2} h^{\prime}+A_{n} F_{n-3}\left(h^{\prime}\right)^{2}-A_{n} F_{n-2} h^{\prime \prime}\right\}-\frac{G_{1} \rho^{\prime}}{\rho^{2}}\left(A_{n}^{\prime} F_{n-1}-A_{n} F_{n-2} h^{\prime}\right) \\
+\frac{G_{1} G_{2}}{\eta_{2} \rho}\left\{A_{n}^{\prime \prime} F_{n}-2 A_{n}^{\prime} F_{n-1} h^{\prime}+A_{n} F_{n-2}\left(h^{\prime}\right)^{2}-A_{n} F_{n-1} h^{\prime \prime}\right\}-\frac{G_{1} G_{2}}{\eta_{2}} \frac{\rho^{\prime}}{\rho^{2}}\left(A_{n}^{\prime} F_{n}-A_{n} F_{n-1} h^{\prime}\right)
\end{gathered}
$$

On simplifying the Eq. (13) using Eq. (11), we get the amplitude function satisfy the equation

$$
2 h^{\prime}(x) A_{n}^{\prime}(x)+\left\{\rho\left(\frac{1}{\eta_{1}}+\frac{1}{\eta_{2}}\right)-(\log \rho)_{, x} h^{\prime}(x)+h^{\prime \prime}(x)\right\} A_{n}(x)=Q_{n},(n=0,1,2 \ldots)
$$

Where,

$$
Q_{n}=A_{n-1}^{\prime \prime}-\left\{\frac{1}{\left(h^{\prime}\right)^{2}}(\log \rho)_{, x}+2 \frac{G_{2}}{\eta_{2}}\right\} A_{n-1}^{\prime}+\frac{G_{2}}{\eta_{2}}\left\{\left(h^{\prime}\right)^{2}(\log \rho)_{, x}-\frac{G_{1}}{\eta_{1}}\left(h^{\prime}\right)^{2}-2 h^{\prime \prime}\right\} A_{n-1}+\frac{G_{2}}{\eta_{2}} A_{n-2}^{\prime \prime}-\frac{G_{2}}{\eta_{2}}(\log \rho)_{, x} A_{n-2}^{\prime}
$$

Which is a linear partial differential equation and its solution is obtained by reducing it into ordinary differential equation using an asymptotic method. The origin of this method is the ray optics and central feature of this method is the motion of rays which are curves or straight lines. The rays are of the fundamental importance because all the functions which make up the various terms of the asymptotic expansion can be shown to satisfy ordinary differential equations along these curves. Thus, this is the one of the method which reduces partial differential equation to ordinary differential equation. Also on using asymptotic method, it is very important to choose the proper signs in the solution of equation (12) so that the direction of the propagation of the wave is taken into consideration.

On integrating Eq. (12), we get

$$
h(x)=h(0) \pm \int_{0}^{x} \frac{d s}{c(s)}
$$

The plus sign shows the wave travelling along positive direction of $\mathrm{x}$-axis and negative sign shows the waves travelling along negative direction of $\mathrm{x}$-axis.

Therefore the solution of Eq. (14) can be obtained as

$$
\begin{gathered}
A_{n}^{\prime}(x)+\frac{1}{2}\left\{\rho c\left(\frac{1}{\eta_{1}}+\frac{1}{\eta_{2}}\right)-(\log \rho)_{, x}+\frac{h^{\prime \prime}(x)}{h^{\prime}(x)}\right\} A_{n}(x)=\frac{c}{2} Q_{n},(n=0,1,2 \ldots) \\
A_{n}^{\prime}(x)+\frac{1}{2}\left\{\rho c\left(\frac{1}{\eta_{1}}+\frac{1}{\eta_{2}}\right)-(\log \rho)_{, x}+(\log h, x), x\right\} A_{n}(x)=\frac{c}{2} Q_{n},(n=0,1,2 \ldots) \\
A_{n}^{\prime}(x)+\left\{m(x)-\frac{1}{2}(\log l(s))_{, x}\right\} A_{n}(x)=\frac{c}{2} Q_{n},(n=0,1,2 \ldots) \\
A_{n}^{\prime}(x)+\frac{1}{2}\left\{\rho c\left(\frac{1}{\eta_{1}}+\frac{1}{\eta_{2}}\right)-(\log \rho c)_{, x}\right\} A_{n}(x)=\frac{c}{2} Q_{n},(n=0,1,2 \ldots)
\end{gathered}
$$


Integrating Factor

$$
e^{\int_{0}^{x}\left\{m(x)-\frac{1}{2}(\log l(s))_{, x}\right\} d s}=\log \left[\frac{l(0)}{l(x)}\right]^{1 / 2} \exp \left(\int_{0}^{x} m(s) d s\right)
$$

And its solution is

$$
A_{n}(x) \log \left[\frac{l(0)}{l(x)}\right]^{1 / 2} \exp \left(\int_{0}^{x} m(s) d s\right)=\frac{1}{2} \int_{0}^{x} c(s) Q_{n}(s) \cdot \log \left[\frac{l(0)}{l(x)}\right]^{1 / 2} \exp \left(\int_{0}^{x} m(s) d s\right) d s+r
$$

Where $r$ is constant of integration.

At $x=0, r=A(0)$

$$
A_{n}(x)=A_{n}(0)\left\{\frac{l(x)}{l(0)}\right\}^{\frac{1}{2}} \exp \left\{-\int_{0}^{x} m(s) d s\right\}+\frac{1}{2}\left\{\frac{l(x)}{l(0)}\right\}^{\frac{1}{2}} \exp \left\{-\int_{0}^{x} m(s) d s\right\} \int_{0}^{x} c(s)\left\{\frac{l(0)}{l(s)}\right\}^{\frac{1}{2}} \exp \left\{+\int_{x}^{z} m(z) d z\right\} \mathrm{Q}_{n}^{ \pm}(s) d s
$$

Which is the expression for wave travelling in the positive direction of $\mathrm{x}$-axis. The expression for wave travelling in positive and negative direction of $\mathrm{x}$-axis is as

$$
A_{n}(x)=A_{n}(0)\left\{\frac{l(x)}{l(0)}\right\}^{\frac{1}{2}} \exp \left\{\mp \int_{0}^{x} m(s) d s\right\} \pm \frac{1}{2} \int_{0}^{x} c(s)\left\{\frac{l(x)}{l(s)}\right\}^{\frac{1}{2}} \exp \left\{ \pm \int_{x}^{z} m(z) d z\right\} \mathrm{Q}_{n}^{ \pm}(s) d s \quad(n=0,1,2 \ldots)
$$

Where,

$$
l(x)=\rho c \quad \text { and } m(x)=\frac{\rho c}{2}\left(\frac{1}{\eta_{1}}+\frac{1}{\eta_{2}}\right)
$$

The upper signs are associated with wave traveling in the positive direction of $x$ and the lower signs are associated with the waves travelling in the negative direction of $x$. At the end $x=0$, the impulse of magnitude $\sigma_{0}$ is suddenly applied and thereafter steadily maintained, that is

$$
\sigma(0, t)=\sigma_{0} H(t)
$$

From Eq. (11) and Eq. (18), we have

$$
\sum_{n=0}^{\infty} A_{n}(0) F_{n}\{t-h(0)\}=\sigma_{0} H(t)
$$

Thus we choose, Moodie (1973)

$$
\begin{aligned}
A_{n}(0) & =\mid \begin{array}{l}
\sigma_{0} \cdots \cdots \cdots \text { if } n=0 \\
0 \cdots \cdots \cdots \text { if } n<0 \text { or } n>0
\end{array} \\
h(0) & =0 \text { and } F_{0}=H(t)
\end{aligned}
$$

The solution of Eq. (9), for the waves travelling in the positive direction of $\mathrm{x}$ is generated by boundary stress Eq. (20), is

$$
\sigma(x, t)=\sum_{n=0}^{\infty} A_{n}(x) \frac{\{t-h(x)\}^{n}}{n !} H\{t-h(x)\}=\sum_{n=0}^{\infty} A_{n}(x) \frac{\{t-h(x)\}^{n}}{n !} H\left\{t-\int_{0}^{x} \frac{d s}{c(s)}\right\}
$$

Where, 


$$
h(x)=\int_{0}^{x} \frac{d s}{c(s)}
$$

Where, $A_{n}(x)$ are given recursively by Eq. (16) (with upper signs) in combination with Eq. (20).

The first-term approximation leads to Eq. (23) as

$$
\sigma(x, t)=\sigma_{0}\left\{\frac{l(x)}{l(0)}\right\}^{\frac{1}{2}} \exp \left\{-\int_{0}^{x} m(s) d s\right\} H\left\{t-\int_{0}^{x} \frac{d s}{c(s)}\right\}
$$

The Eq. (24) represents a transient stress wave which starts from the end ' $x=0$ ' with amplitude ' $\sigma_{0}$ ' and moves in the positive direction of ' $x$ ' with velocity $c(x)$. Hence, it is modulated by the factor

$$
\left\{\frac{l(x)}{l(0)}\right\}^{\frac{1}{2}} \exp \left\{-\int_{0}^{x} m(s) d s\right\}
$$

Further terms in the approximate solution may be obtained recursively from Eq. (19)

\section{Viscoelastic Model Applied to a Particular Case}

For the sake of concreteness and for studying the qualitative effect of non-homogeneity on the longitudinal wave propagation in non-homogeneous four parameter viscoelastic rods, it is assumed that density ' $\rho$ ', rigidity ' $G$ ' and viscosity ' $\eta$ ' of the specimen i.e. rod are space dependent and obey the harmonic laws

$$
\rho=\rho_{0} e^{2 i \alpha_{1} x}, G=G_{0} e^{2 i \alpha_{2} x}, \eta=\eta_{0} e^{2 i \alpha_{3} x}
$$

If, $\quad \alpha_{1} \geq \alpha_{2} \geq \alpha_{3}$ i.e. density $\geq$ rigidity $\geq$ viscosity

\section{Case-1}

When, $\alpha_{1}=\alpha_{2}=\alpha_{3}$, then from Eq. (26), we get

$$
\rho=\rho_{0} e^{2 i \alpha x}, G=G_{0} e^{2 i \alpha x}, \eta=\eta_{0} e^{2 i \alpha x}
$$

Therefore, from Eikonal equation of geometric optics

$$
\begin{aligned}
\left(\frac{d h(x)}{d x}\right)^{2} & =\frac{\rho}{G_{1}}=\frac{\rho_{0} e^{2 i \alpha x}}{G_{10} e^{2 i \alpha x}}=\frac{\rho_{0}}{G_{10}}=\frac{1}{c_{0}^{2}}=\text { constant. } \\
c_{0} & =\sqrt{\frac{G_{10}}{\rho_{0}}}
\end{aligned}
$$

Since, the harmonic variation of modulus of rigidity $G$ and density $\rho$ is similar, therefore sound speed is constant i.e. nonhomogeneous has no effect on speed and phase of the wave is given $h(x)=\frac{x}{c_{0}}$. So it becomes the case of semi nonhomogeneous medium (a medium when characteristics are space dependent while the speed is independent of space variable).

The amplitude function $A_{n}(x)$ satisfies the equation

$$
2 h^{\prime}(x) A_{n}^{\prime}(x)+\left\{\rho_{0}\left(\frac{1}{\eta_{10}}+\frac{1}{\eta_{20}}\right)-2 i \alpha h^{\prime}(x)\right\} A_{n}(x)=Q_{n}^{\prime}, \quad(n=0,1,2 \ldots \ldots . .)
$$

Where, 
$Q_{n}^{\prime}=A_{n-1}^{\prime \prime}-\left\{\frac{1}{\left(h^{\prime}\right)^{2}} 2 i \alpha+2 \frac{G_{20}}{\eta_{20}}\right\} A_{n-1}^{\prime}+\frac{G_{2}}{\eta_{2}}\left\{\left(h^{\prime}\right)^{2}(2 i \alpha)-\frac{G_{10}}{\eta_{10}}\left(h^{\prime}\right)^{2}\right\} A_{n-1}+\frac{G_{20}}{\eta_{20}} A_{n-2}^{\prime \prime}-\frac{G_{20}}{\eta_{20}}(2 i \alpha) A_{n-2}^{\prime}$

As the amplitude function is given by Eq.(15), For this case

$$
\begin{gathered}
l(x)=\sqrt{\rho_{0} G_{10}} e^{2 i \alpha x} \\
m(x)=\frac{\sqrt{\rho_{0} G_{10}}}{2}\left(\frac{1}{\eta_{10}}+\frac{1}{\eta_{20}}\right)=m_{0} \\
\int_{0}^{x} m(x) d x=m_{0} x
\end{gathered}
$$

Hence,

$$
A_{n}(x)=A_{n}(0) e^{i \alpha x} \exp \left\{-m_{0} x\right\} \pm \frac{1}{2} c_{0} \mathrm{e}^{i \alpha x} \exp \left\{ \pm \int_{x}^{s} m_{0} d z\right\} Q_{n}^{\prime \pm}(s) d s
$$

For this case the value of first term approximation, the stress function is given by

$$
\begin{aligned}
& \sigma(x, t)=\sigma_{0} e^{i \alpha x} \exp \left\{-\int_{0}^{x} m_{0} d s\right\} H\{t-h(x)\} \\
& \sigma(x, t)=\sigma^{\prime}+i \sigma^{\prime \prime}
\end{aligned}
$$

Where $\sigma^{\prime}$ and $\sigma^{\prime \prime}$ represents the real and imaginary parts respectively when $\rho, G, \eta$, obeys harmonic laws.

The expression for the wave front at $t=h(x)$ is as

$$
\sigma(x, t)=\sigma_{0}(\cos \alpha x) \exp \left\{-m_{0} x\right\}+i \sigma_{0}(\sin \alpha x) \exp \left\{-m_{0} x\right\}
$$

The progressive harmonic wave which starts from the end $x=0$ with amplitude $\sigma_{0}$ and moves with constant velocity $c_{0}=\sqrt{\frac{G_{10}}{\rho_{0}}}$ in the positive direction of $x$ is modulated by the factor $\sigma_{0}(\cos \alpha x) \exp \left\{-m_{0} x\right\}$ and attenuation by the factor $\sigma_{0}(\sin \alpha x) \exp \left\{-m_{0} x\right\}$.

\section{Case II}

$\alpha_{1}>\alpha_{2}>\alpha_{3}$ i.e. density $>$ rigidity $>$ viscosity, then from Eq. (25), we get

$$
\rho=\rho_{0} e^{2 i \alpha_{1} x}, G=G_{0} e^{2 i \alpha_{2} x}, \eta=\eta_{0} e^{2 i \alpha_{3} x}
$$

From Eikonal equation of geometric optics

$$
\left(\frac{d h(x)}{d x}\right)^{2}=\frac{\rho}{G_{1}}=\frac{\rho_{0} e^{2 i \alpha_{1} x}}{G_{10} e^{2 i \alpha_{2} x}}=\frac{\rho_{0}}{G_{10}} e^{2 i\left(\alpha_{1}-\alpha_{2}\right) x}=\frac{1}{c^{2}}, \text { Here, } c=\sqrt{\frac{G_{10}}{\rho_{0}}} e^{i\left(\alpha_{2}-\alpha_{1}\right) x}
$$

The amplitude function $A_{n}(x)$ satisfies the equation

$$
2 h^{\prime}(x) A_{n}^{\prime}(x)+\left\{\rho_{0} e^{2 i\left(\alpha_{1}-\alpha_{3) x}\right.}\left(\frac{1}{\eta_{10}}+\frac{1}{\eta_{20}}\right)-2 i \alpha_{1} h^{\prime}(x)+h^{\prime \prime}(x)\right\} A_{n}(x)=Q_{n}^{\prime \prime},(n=0,1,2 \ldots \ldots . .)
$$


Where,

$$
Q_{n}^{\prime \prime}=A_{n-1}^{\prime \prime}-\left\{\frac{1}{\left(h^{\prime}\right)^{2}} 2 i \alpha_{1}+2 K\right\} A_{n-1}^{\prime}-K_{1}\left\{\left(h^{\prime}\right)^{2}\left(2 i \alpha_{1}\right)-\frac{G_{10}}{\eta_{10}} e^{2 i\left(\alpha_{1}-\alpha_{3}\right) x}\left(h^{\prime}\right)^{2}-2 h^{\prime \prime}\right\} A_{n-1}+K_{1} A_{n-2}^{\prime \prime}-2 K_{1} i \alpha_{1} A_{n-2}^{\prime}(n=0,1,2 \ldots \ldots \ldots .)
$$

and $K_{1}=e^{2 i\left(\alpha_{2}-\alpha_{3}\right) x} \frac{G_{10}}{G_{20}}$. Amplitude function $A_{n}(x)$ is given by Eq.(16).

For this case

$$
\begin{gathered}
l(x)=\sqrt{G_{10} \rho_{0}} e^{i\left(\alpha_{1}+\alpha_{2}\right) x}=l_{1}(x) \text { and } m(x)=\frac{\sqrt{G_{10} \rho_{0}}}{2} e^{i\left(\alpha_{1}+\alpha_{2}-2 \alpha_{3}\right) x}\left\{\frac{1}{\eta_{10}}+\frac{1}{\eta_{20}}\right\}=m_{1}(x) \\
\int_{0}^{x} m(x) d x=\frac{\frac{\sqrt{G_{10} \rho_{0}}}{2}\left\{\frac{1}{\eta_{10}}+\frac{1}{\eta_{20}}\right\}}{i\left(\alpha_{1}+\alpha_{2}-2 \alpha_{3}\right)}\left\{e^{i\left(\alpha_{1}+\alpha_{2}-2 \alpha_{3}\right)}-1\right\}, \frac{l(x)}{l(0)}=\left\{e^{i\left(\alpha_{1}+\alpha_{2}\right) x}\right\}=\frac{l_{1}(x)}{l_{1}(0)}
\end{gathered}
$$

Therefore

$$
A_{n}(x)=A_{n}(0)\left\{e^{i\left(\alpha_{1}+\alpha_{2}\right) x}\right\}^{\frac{1}{2}} \exp \left\{\mp \int_{0}^{x} m_{1}(s) d s\right\} \pm \frac{1}{2} \int_{0}^{x} c(s)\left\{\frac{l_{1}(x)}{l_{1}(s)}\right\}^{\frac{1}{2}} \exp \left\{ \pm \int_{x}^{z} m_{1}(z) d z\right\} Q_{n}^{\prime \prime}(s) d s
$$

For this case the value of first term approximation, the stress function is given by

$$
\begin{gathered}
\sigma(x, t)=\sigma_{0}\left(e^{i r_{1} x}\right)^{\frac{1}{2}} \exp \left\{i p_{2}\left(e^{i r_{2} x}-1\right)\right\} H\{t-h(x)\} \\
\sigma(x, t)=\sigma_{0} \exp \left(-p_{2} \sin r_{2} x\right)\left(\cos \frac{r_{1}}{2} x+i \sin \frac{r_{1}}{2} x\right)\left\{\cos p_{2}\left(\cos r_{2} x-1\right)+i \sin p_{2}\left(\cos r_{2} x-1\right)\right\} H\{t-h(x)\} \\
\sigma(x, t)=\sigma^{\prime}+i \sigma^{\prime \prime}
\end{gathered}
$$

The equation of wave front at $t=h(x)$ is given by

$$
\begin{gathered}
\sigma(x, t)=\sigma_{0} \exp \left(-p_{2} \sin r_{2} x\right)\left[\cos \left\{\frac{r_{1}}{2} x+p_{2}\left(\cos r_{2} x-1\right)\right\}\right]+i \sigma_{0} \exp \left(-p_{2} \sin r_{2} x\right)\left[\sin \left\{\frac{r_{1}}{2} x+p_{2}\left(\cos r_{2} x-1\right)\right\}\right] \\
\sigma(x, t)=\sigma_{0} \exp \left(-p_{2} \sin r_{2} x\right)\left[\cos \left\{\frac{r_{1}}{2} x+p_{2}\left(\cos r_{2} x-1\right)\right\}\right] H\{t-h(x)\} \\
+i \sigma_{0} \exp \left(-p_{2} \sin r_{2} x\right)\left[\sin \left\{\frac{r_{1}}{2} x+p_{2}\left(\cos r_{2} x-1\right)\right\}\right] H\{t-h(x)\}
\end{gathered}
$$

The progressive harmonic wave which starts from the end $x=0$ with amplitude $\sigma_{0}$ and moves with constant velocity $C=\sqrt{\frac{G_{10}}{\rho_{0}}} e^{i\left(\alpha_{2}-\alpha_{1}\right) x} \quad$ in the positive direction of ' $X$ ' is modulated by the factor $\sigma_{0} \exp \left(-p_{2} \sin r_{2} x\right)\left[\cos \left\{\frac{r_{1}}{2} x+p_{2}\left(\cos r_{2} x-1\right)\right\}\right]$.

The attenuation factor is given by 
$\sigma_{0} \exp \left(-p_{2} \sin r_{2} x\right)\left[\sin \left\{\frac{r_{1}}{2} x+p_{2}\left(\cos r_{2} x-1\right)\right\}\right]$.

Where

\section{Numerical Analysis}

$$
r_{1}=\alpha_{1}+\alpha_{2}, r_{2}=r_{1}-2 \alpha_{3}, \frac{\sqrt{G_{10} \rho_{0}}}{2}\left\{\frac{1}{\eta_{10}}+\frac{1}{\eta_{20}}\right\}=p_{1}, \frac{p_{1}}{r_{2}}=p_{2} .
$$

Here, all the mechanical properties obey harmonic laws. As $x$ lies between $0 \leq x \leq \infty$ and also $x$ depends upon $\alpha$.Two distinct cases are considered for taking $\alpha<1$ and $\alpha>1$.

Let the parameters are as

Table 1. Material Parameters

\begin{tabular}{cccc}
$\rho_{0}$ & $G_{10}$ & $\eta_{10}$ & $\eta_{20}$ \\
\hline 1.8 & 1.6 & 1.2 & 1.3 \\
\hline
\end{tabular}

Case: 1

For $\alpha<1$, Let $\alpha=1 / 2, m_{0}=8.12$

The equation of wave front at $t=h(x)=1.06 x$ is as

$$
\frac{\sigma}{\sigma_{0}}=\left(\cos \frac{x}{2}\right) \exp \{-8.12 x\}+i\left(\sin \frac{x}{2}\right) \exp \{-8.12 x\}
$$

The wave is modulated by the factor

$$
\left(\cos \frac{x}{2}\right) \exp \{-8.12 x\}
$$

and attenuated by the factor

$$
\left(\sin \frac{x}{2}\right) \exp \{-8.12 x\}
$$

For $\alpha>1$, Let $\alpha=2, m_{0}=8.12$

The equation of wave front at $t=h(x)=1.06 x$ is as

$$
\frac{\sigma}{\sigma_{0}}=(\cos 2 x) \exp \{-8.12 x\}+i \sigma_{0}(\sin 2 x) \exp \{-8.12 x\}
$$

The wave is modulated by the factor

$$
(\cos 2 x) \exp \{-8.12 x\}
$$

And attenuated by the factor

$$
(\sin 2 x) \exp \{-8.12 x\}
$$

Case: 2

For non-homogeneous case 
Table 2. Material Parameters

\begin{tabular}{llllllll}
\hline & $\alpha_{1}$ & $\alpha_{2}$ & $\alpha_{3}$ & $r_{1}$ & $r_{2}$ & $p_{1}$ & $p_{2}$ \\
\hline$\alpha<1$ & $1 / 2$ & $1 / 4$ & $1 / 6$ & $3 / 4$ & $1 / 2$ & 8.12 & 16.24 \\
\hline$\alpha>1$ & 8 & 4 & 2 & 12 & 8 & 8.12 & 1.015 \\
\hline
\end{tabular}

For $\alpha<1$

The equation of wave front at $t=h(x)=2.12 \sin \frac{x}{2}-2.12 i\left(\cos \frac{x}{2}-1\right)$ is as

$$
\frac{\sigma}{\sigma_{0}}=\exp \left(-16.24 \sin \frac{x}{2}\right)\left[\cos \left\{\frac{3}{8} x+16.24\left(\cos \frac{x}{2}-1\right)\right\}\right]+i \exp \left(-16.24 \sin \frac{x}{2}\right)\left[\sin \left\{\frac{3}{8} x+16.24\left(\cos \frac{x}{2}-1\right)\right\}\right]
$$

The modulated factor is given by

$$
\frac{\sigma}{\sigma_{0}}=\exp \left(-16.24 \sin \frac{x}{2}\right)\left[\cos \left\{\frac{3}{8} x+16.24\left(\cos \frac{x}{2}-1\right)\right\}\right]
$$

The attenuation is given by the factor

$$
\exp \left(-16.24 \sin \frac{x}{2}\right)\left[\sin \left\{\frac{3}{8} x+16.24\left(\cos \frac{x}{2}-1\right)\right\}\right]
$$

For $\alpha>1$

The equation of wave front at $t=h(x)=0.26 \sin \frac{x}{2}-i(0.26)(\cos 4 x-1)$ is as

$$
\frac{\sigma}{\sigma_{0}}=\exp (-1.015 \sin 8 x)[\cos \{6 x+(1.015)(\cos 8 x-1)\}]+i \exp (-1.015 \sin 8 x)[\sin \{6 x+1.015(\cos 8 x-1)\}]
$$

The modulated factor is given by

$$
\exp (-1.015 \sin 8 x)[\cos \{6 x+(1.015)(\cos 8 x-1)\}]
$$

The attenuation is given by the factor

$$
\exp (-1.015 \sin 8 x)[\sin \{6 x+1.015(\cos 8 x-1)\}]
$$

To see qualitative effect of non-homogeneity on the harmonic wave propagation in non-homogeneous four parameter viscoelastic rods, the various graphs are plotted between $\frac{\sigma}{\sigma_{0}}$ and $x$. For the semi homogeneous cases Figure (2) represents the plot for Eq.(43) and Figure (3) represents the plot for Eq.(44).It shows that there is slight variation in the wave in the neighborhood of $x=0$ As $x$ increases the wave becomes constant. Figure (2) represents the wave in progress and Figure (3) represents its attenuation. It is also observed that for $\alpha<1$, the wave progression is near the origin and not along the $x$ apart. The Figure (4) and (5) also represents the similar result for $\alpha>1$. So it can be concluded that the value of $\alpha$ does not impact more in the semi homogeneous case and also that the wave progression is at near the starting point only. For the non-homogeneous cases, Figure (6) and Figure (7) represents the plot for Eq.(48) and Eq.(49) respectively. Figure (8) and Figure (9) represents the plot for Eq. (51) and Eq. (52) respectively for $\alpha<1$ and for $\alpha>1$ harmonic wave is in progress but with unequal interval of times. Thus the effect of non-homogeneity is clearly observed for the harmonic waves in non-homogeneous four parameter viscoelastic model. 


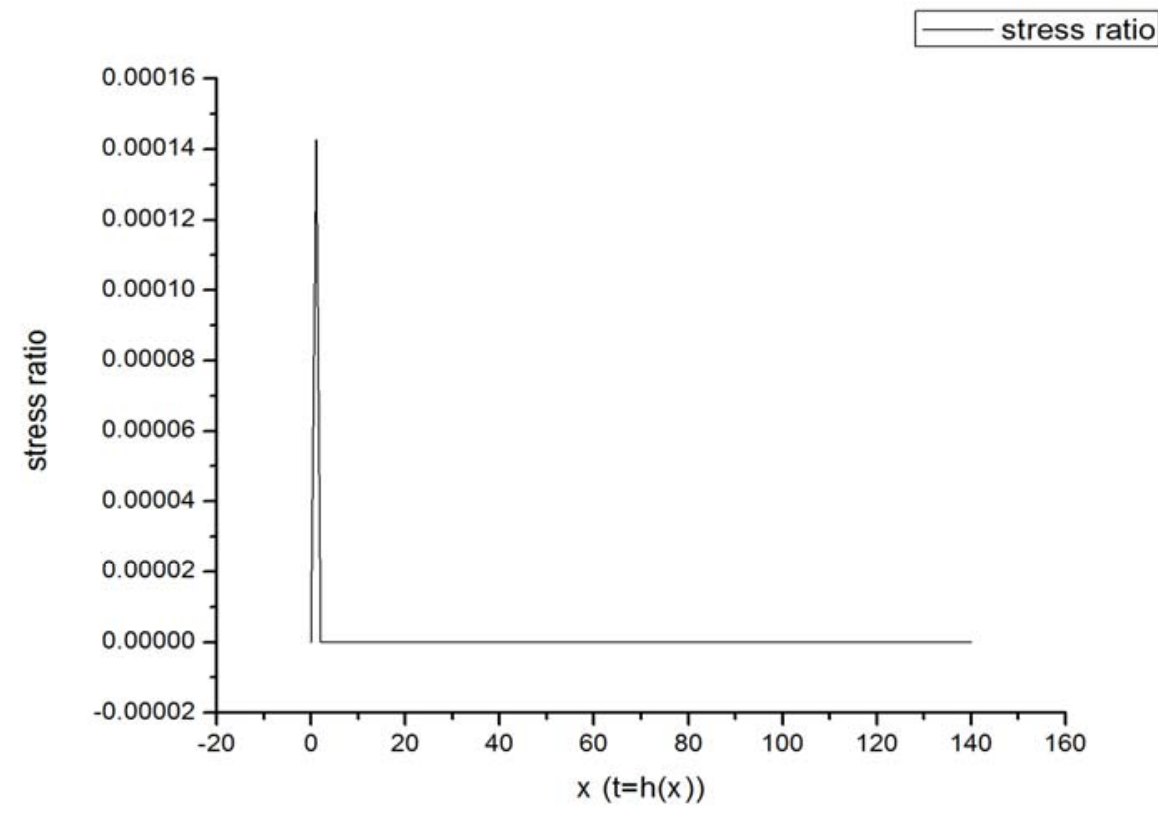

Figure 2. Wave in progress in homogeneous case for $\alpha<1$

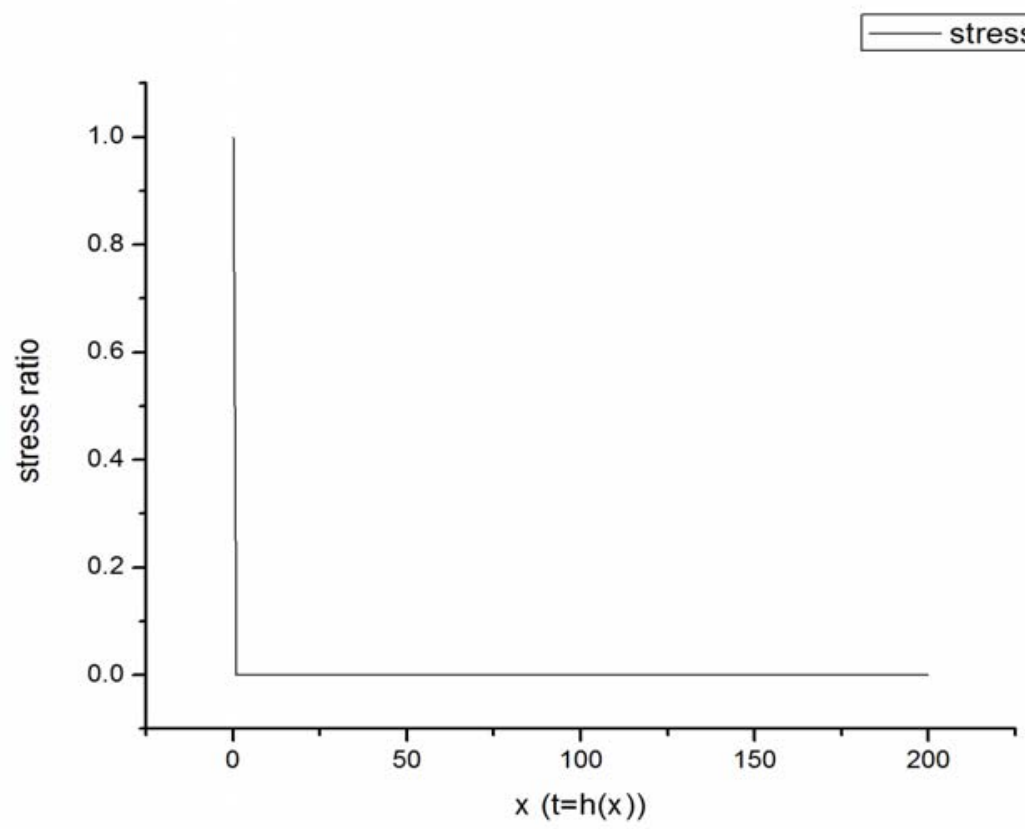

Figure 3. Attenuation of the wave in homogeneous case for $\alpha<1$ 


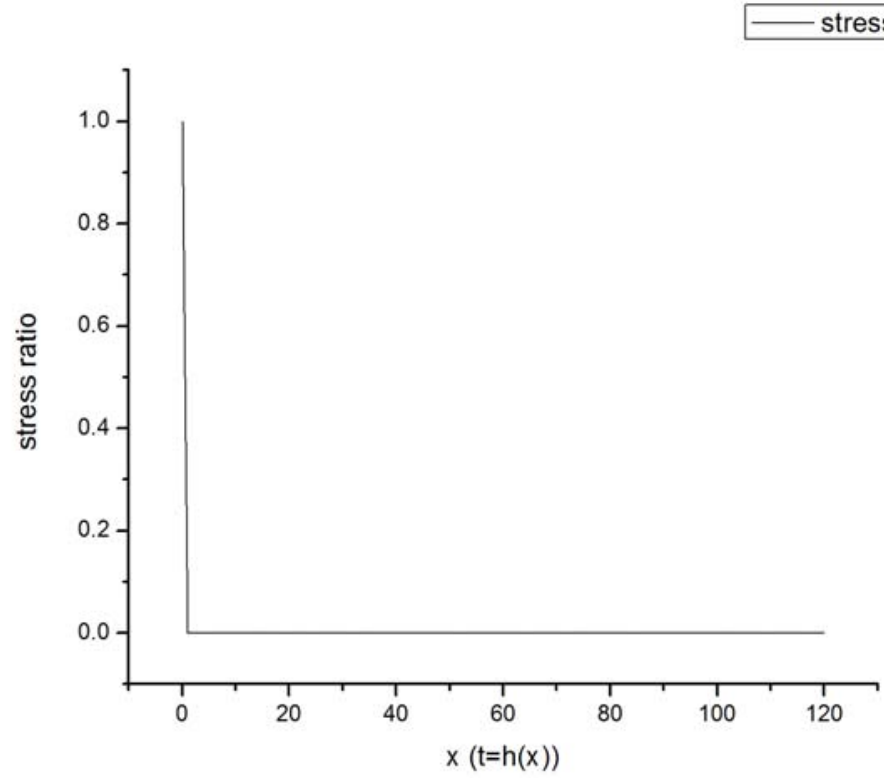

Figure 4. Wave in progress in homogeneous case for $\alpha>1$

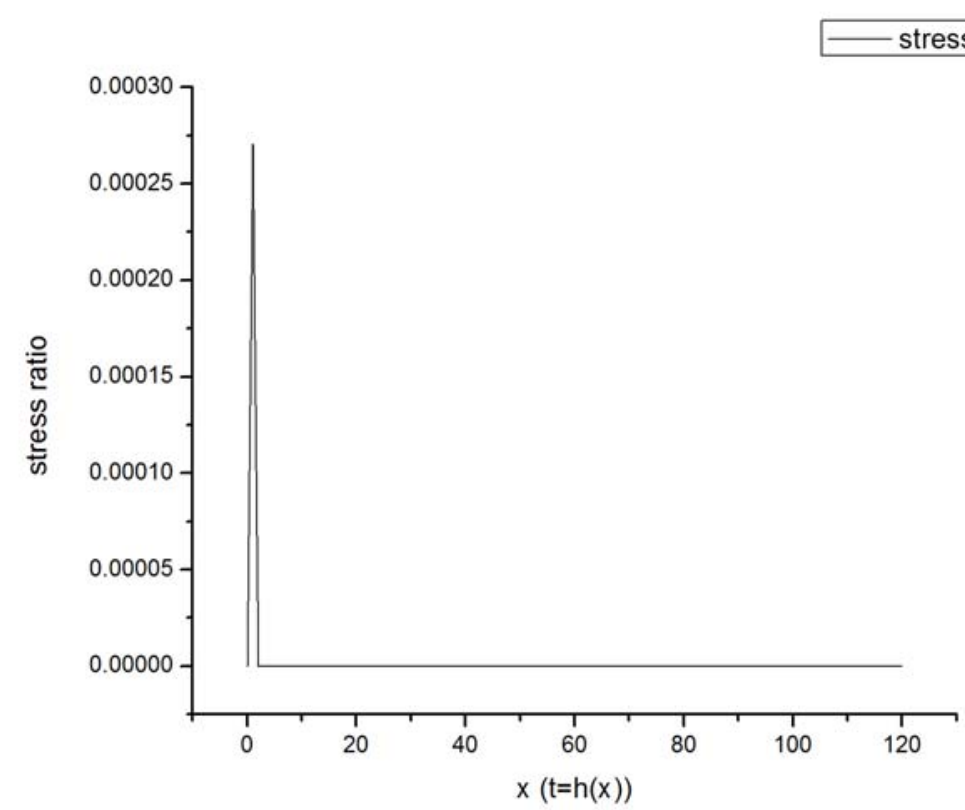

Figure 5. Attenuation of the wave in homogeneous case for $\alpha>1$ 


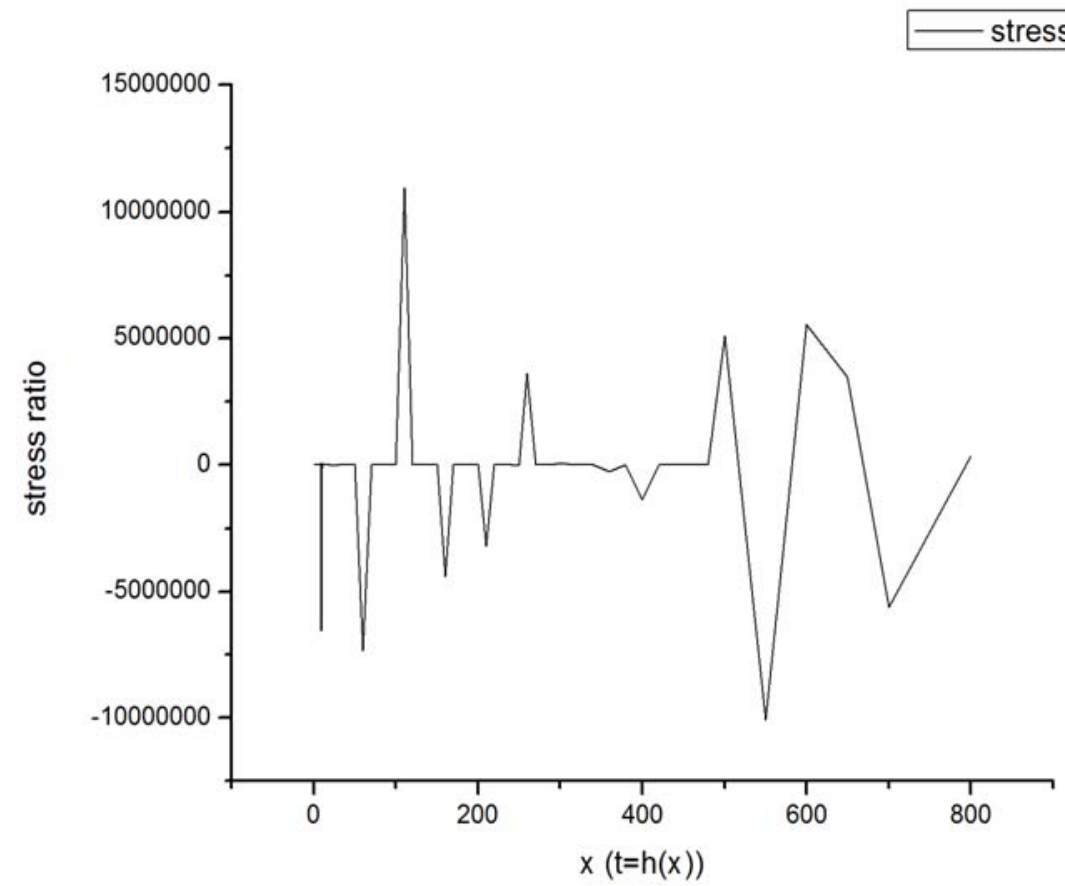

Figure 6. Wave in progress in non-homogeneous case for $\alpha<1$

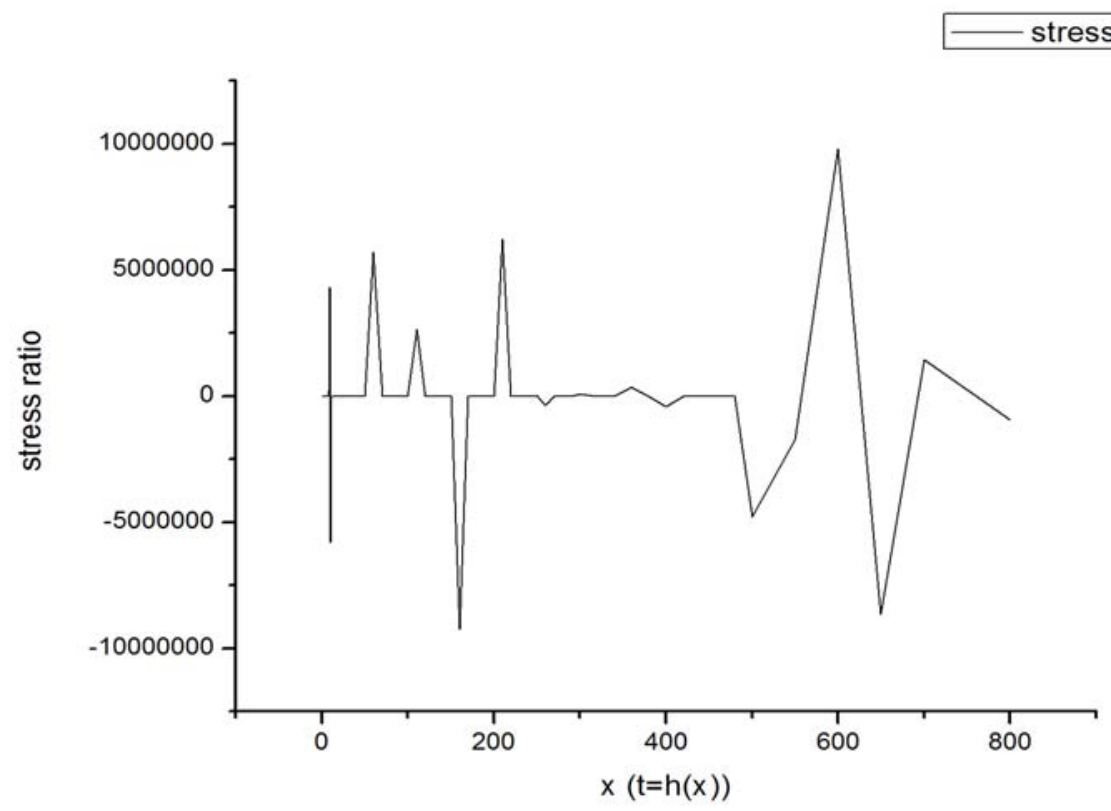

Figure 7. Attenuation of the wave in non-homogeneous case for $\alpha<1$ 


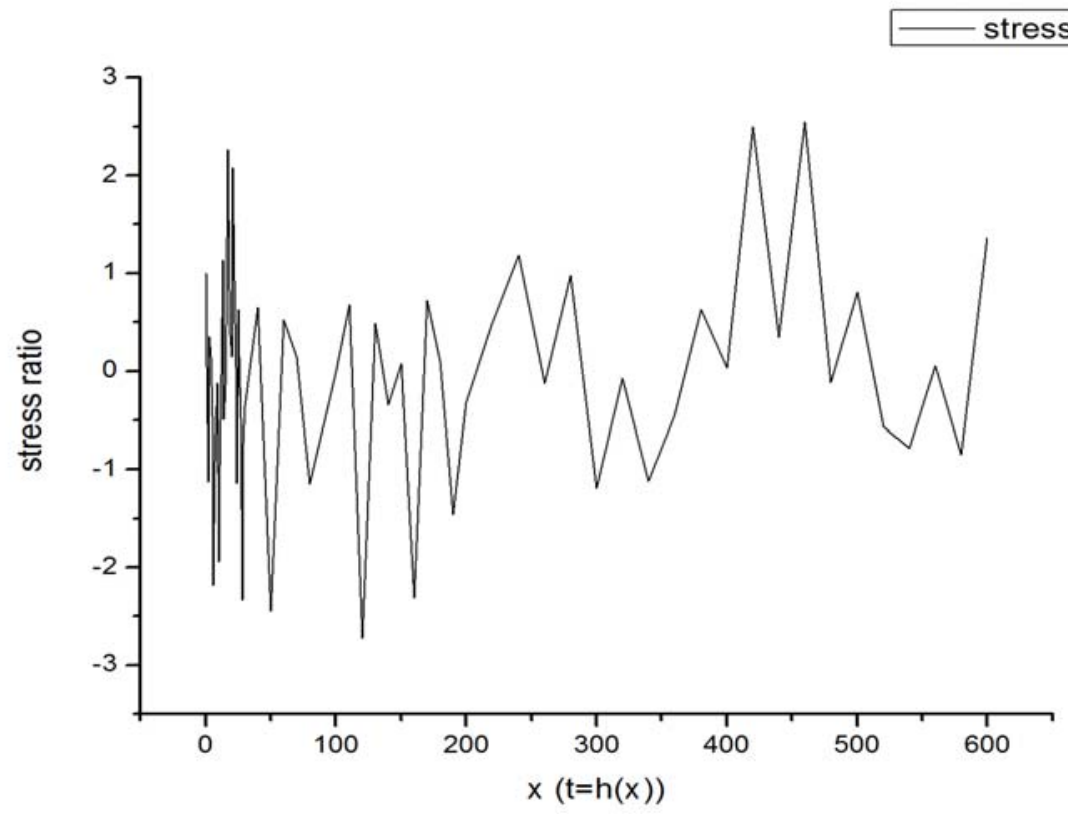

Figure 8. Wave in progress in non-homogeneous case for $\alpha>1$

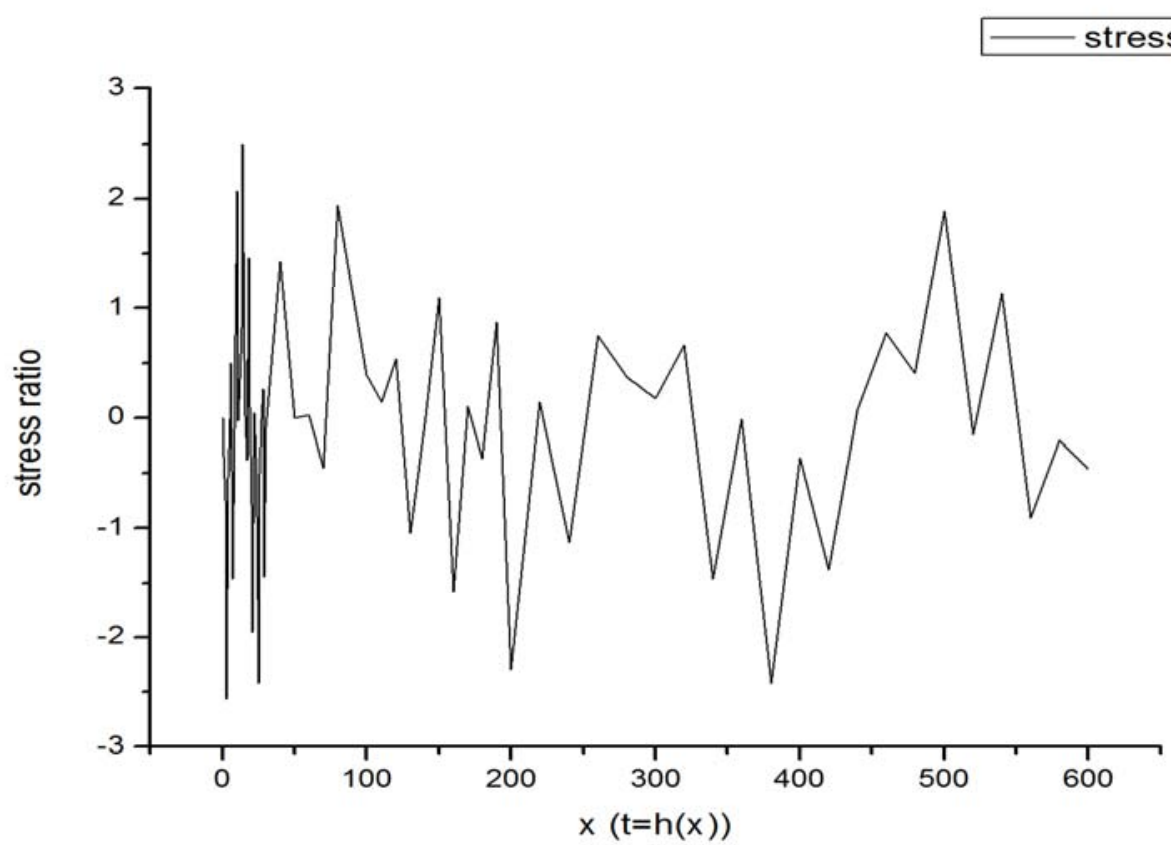

Figure 9. Attenuation of the wave for non-homogeneous case for $\alpha>1$

\section{Dynamic response of four parameter viscoelastic model}

The following values of strain are taken for the dynamical response of four parameter model

$$
\sigma=G^{*} a_{0} e^{i w t}, a=a_{0} e^{i w t}
$$


From Eq. (1) and Eq. (53), we get

$$
\left\{-\omega^{2}+\left(\frac{G_{1}}{\eta_{2}}+\frac{G_{2}}{\eta_{2}}+\frac{G_{1}}{\eta_{1}}\right) i \omega+\frac{G_{1} G_{2}}{\eta_{1} \eta_{2}}\right\} G^{*}=G_{1}\left\{-\omega^{2}+\left(\frac{G_{1} G_{2}}{\eta_{2}}\right) i \omega\right\}
$$

On simplifying Eq. (54), we get

$$
G^{*}=\frac{G_{1}\left(-\omega^{2}+R_{8} i \omega\right)\left\{\left(R_{9}-\omega^{2}\right)-\left(R_{5}+R_{6}+R_{7}\right) i \omega\right\}}{A_{2}}
$$

where, $A_{2}=\left(R_{9}-\omega^{2}\right)^{2}+\left(R_{5}+R_{6}+R_{7}\right)^{2} \omega$

$G^{*}$ can be written in terms of real and imaginary parts

$$
\begin{gathered}
G^{*}=\frac{G_{1}\left[\left(\omega^{2}-R_{9}\right) \omega^{2}+\left\{R_{8}\left(R_{5}+R_{6}+R_{7}\right)\right\} \omega^{2}\right]+i\left\{R_{8}\left(R_{9}-\omega^{2}\right) \omega+R_{8}\left(R_{5}+R_{6}+R_{7}\right) \omega^{3}\right\}}{A_{2}} \\
\text { or } G^{*}=G^{\prime}+G^{\prime \prime} \\
G^{\prime}=\frac{G_{1}\left[\left(\omega^{2}-R_{9}\right) \omega^{2}+\left\{R_{8}\left(R_{5}+R_{6}+R_{7}\right)\right\} \omega^{2}\right]}{A_{2}}, \\
G^{\prime \prime}=\frac{\left\{R_{8}\left(R_{9}-\omega^{2}\right) \omega+R_{8}\left(R_{5}+R_{6}+R_{7}\right) \omega^{3}\right\}}{\left(R_{9}-\omega^{2}\right)^{2}+\left(R_{5}+R_{6}+R_{7}\right)^{2} \omega} .
\end{gathered}
$$

The loss tangent is,

$$
\tan \delta=\frac{G^{\prime \prime}}{G^{\prime}}
$$

From Eq. (58), Eq. (59) and Eq. (60), we get

$$
\tan \delta=\frac{R_{8}\left(R_{9}-\omega^{2}\right)+R_{8}\left(R_{5}+R_{6}+R_{7}\right) \omega}{\left(\omega^{2}-R_{9}\right)+\left\{R_{8}\left(R_{5}+R_{6}+R_{7}\right)\right\}}
$$

The behavior of the model has been studied numerically as well as graphically, the rheological responses are discussed by plotting a graph between $G^{\prime \prime}$ and $\omega$ and $G^{\prime}$ verses $\omega$ for four parameter model.

Four parameter model, we have

$$
G^{\prime \prime}=\frac{\left\{R_{8}\left(R_{9}-\omega^{2}\right) \omega+\left\{R_{8}\left(R_{5}+R_{6}+R_{7}\right)\right\} \omega^{3}\right\}}{\left(R_{9}-\omega^{2}\right)^{2}+\left(R_{5}+R_{6}+R_{7}\right)^{2} \omega} .
$$


The values of the parameters for studying the rheological response are

$$
G_{1}=1.0, \quad G_{2}=1.10, \quad \eta_{1}=0.1, \quad \eta_{2}=0.2, \quad R_{5}=5, \quad R_{6}=5.5, \quad R_{7}=10, \quad R_{8}=5.5, \quad R_{9}=55
$$

Now,

$$
G^{\prime}=\frac{G_{1}\left[\left(\omega^{2}-R_{9}\right) \omega^{2}+\left\{R_{8}\left(R_{5}+R_{6}+R_{7}\right)\right\} \omega^{2}\right]}{\left(R_{9}-\omega^{2}\right)^{2}+\left(R_{5}+R_{6}+R_{7}\right)^{2} \omega}
$$

A graph between $G^{\prime}$ verses $\omega$ is plotted by taking above equation. The parameters taken for this case are the same.

The Figure (5-6), shows that as the value of $\omega$ increases both $G^{\prime \prime}$ and $G^{\prime}$ decreases, but the value of $G^{\prime}$ becomes constant after $\omega=55$ for four parameter model.

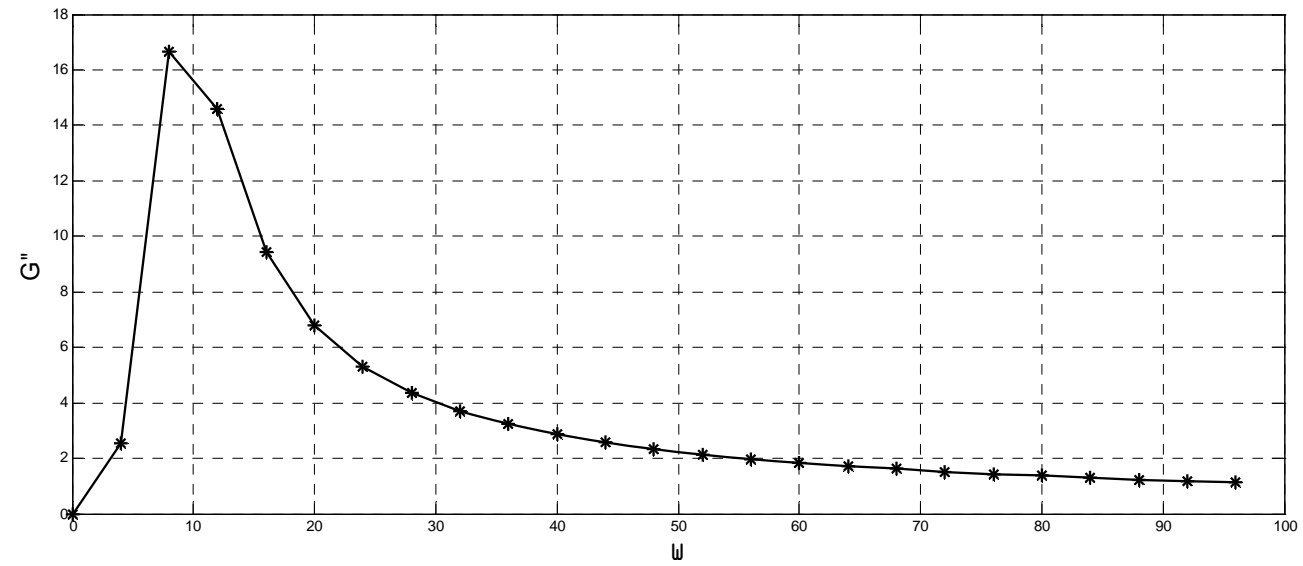

Figure 10. Variation of $G^{\prime \prime}$ verses $\omega$ for four parameter model

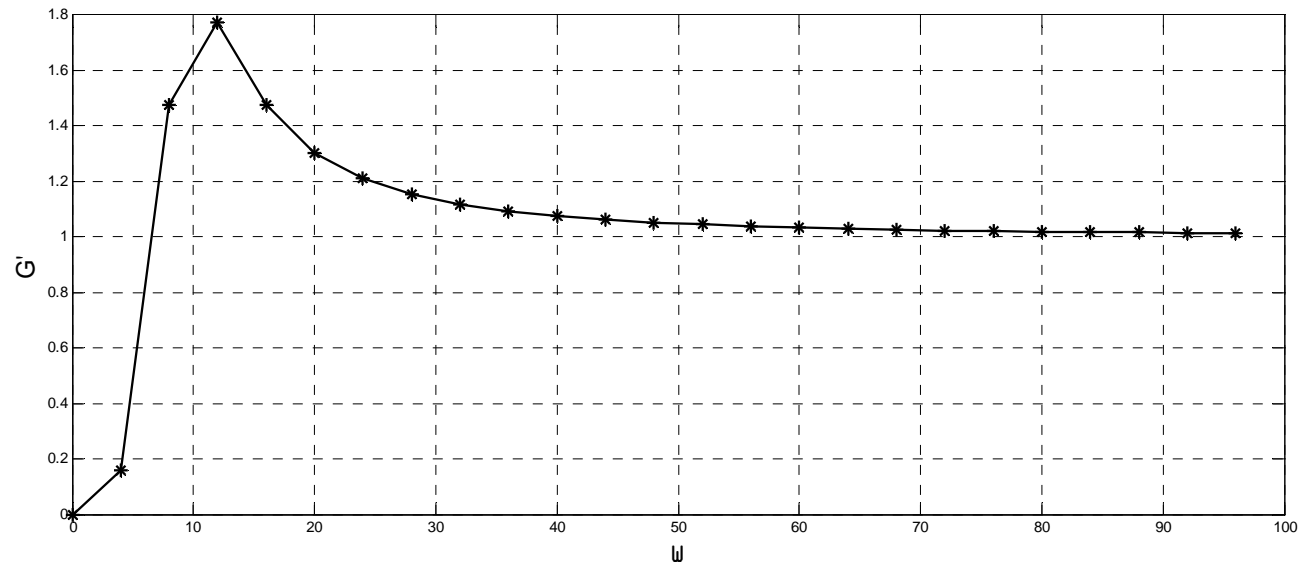

Figure 11. Variation of $G^{\prime}$ verses $\omega$ for four parameter model

\section{Conclusions}

o It can be concluded that the four parameter model possesses an excellent potential for proper representation of the time dependent behavior of a viscoelastic medium subjected to loading and unloading. The viscous strains due to a constant stress are found to increase linearly with time. 
o Moreover, after the removal of stress, the viscous strain is found to remain constant with time. The use of four parameters is mostly restricted in the field of rock mechanics. Thus, models can be used in determining the time-dependent behavior of a viscoelastic medium.

o When the density, rigidity and viscosity all are equal for the first material specimen, the sound speed is constant i.e. nonhomogeneous has no effect on speed and phase of the wave is given $h(x)=\frac{x}{c_{0}}$. So it becomes the case of semi nonhomogeneous medium (a medium when characteristics are space dependent while the speed is independent of space variable). The longitudinal speed will be equal to $C=\sqrt{\frac{G_{10}}{\rho_{0}}}$.

o When the density, rigidity and viscosity are not equal for the second material specimen, the speed of sound varies exponential as $C=\sqrt{\frac{G_{10}}{\rho_{0}}} e^{i\left(\alpha_{2}-\alpha_{1}\right) x}$

\section{Acknowledgements}

The authors are thankful to the referees for their valuable comments.

\section{References}

Batra R. C., 1998. Linear Constitutive Relations in Isotropic Finite Elasticity, J. Elasticity, Vol. 51, pp. 243-245.

Bert, C. W. and Egle D. M., 1969. Wave Propagation in a Finite Length Bar with Variable Area of Cross-section, J. Appl. Mech. (ASME), Vol. 36, pp. 908-909.

Breuer and Shlomo, 1970. Minimum principles in linear viscoelasticity, J. Mecanique, 9, pp. 267-277.

Biot M. A. 1940. Influence of Initial Stress on Elastic Waves, Journal of Applied Physics, Vol.11. No. 8, pp. 522-530.

Carslaw H. S. and Jaeger J. C., 1963. Operational Methods in Applied Math, Second Ed., Dover Pub, New York.

Chandra B., 1980. Radial vibrations of thin circular if variable modulus of elasticity and variable density with a central hole, Indian Journal of Theoretical Physics, Vol. 28, No.3, pp. 281-286.

Christensen R. M., 1971.Theory of Viscoelasticity, Academic Press.

Friedlander F. G. 1947. Simple progressive solutions of the wave equation, Proc. Camb. Phil. Soc., Vol. 43, pp. 360-73.

Gaiduk S.I. 1971. A certain problem concerning the longitudinal oscillations of a composite viscoelastic relaxing rod, Vesci. Akad. Nauk B.S.C.R. Ser, Fiz. Mat. Nauk, pp. 56-63.

Kakar R., Kaur K. and Gupta K.C. 2012. Analysis of five-parameter viscoelastic model under dynamic loading, J. Acad. Indus. Res., Vol.1. No.7, pp. 419-426.

Kakar, R. and Kaur, K. 2013. Mathematical analysis of five parameter model on the propagation of cylindrical shear waves in nonhomogeneous viscoelastic media, International Journal of Physical and Mathematical Sciences, Vol. 4. No. 1, pp. 45-52.

Kakar R., Kaur K. and Gupta K.C., 2013. Study of Viscoelastic Model for Longitudinal Wave Propagation in a non-homogeneous Viscoelastic Filament, Int. J. Pure Appl. Sci. Technol., Vol. 1. No.1, pp. 1-11.

Kakar R. Kaur K. and Gupta K.C. 2013. Study of viscoelastic model for harmonic waves in nonhomogeneous viscoelastic filaments, Interactions and Multiscale Mechanics, Vol. 6. No. 1, pp. 26-45.

Kaur K., Kakar R., Kakar S. and Gupta, K.C., 2013. Applicability of four parameter viscoelastic model for longitudinal wave propagation in non-homogeneous rods, International Journal of Engineering Science and Technology, Vol. 5. No. 1, pp. 75-90.

Kaur K., Kakar, R. and Gupta K.C., 2012. A dynamic non-linear viscoelastic model, International Journal of Engineering Science and Technology, Vol. 4, No. 12, pp. 4780-4787.

Karl F. C. and Keller J. B., 1959, Elastic waves propagation in homogeneous and inhomogeneous media, Journal of Acoustical Society America, Vol. 31, pp. 694-705.

Keolsky, H., 1966. The Propagation of stress waves in viscoelastic solids, Appl. Mech. Surveys.

Krickeja S.S., 1971. Mathematical considerations of the problem of longitudinal oscillations of an elasto-viscous nonhomogeneous rod, Izv. Vyss. Ucebin, Zavecl Mathematica, Vol. 197, No. 4, pp. 37-41.

Miklowitz J., 1966. Elastic wave propagation, Applied Mechanics Survey, Spartan Books.

Mirsky I., 1965. Wave propagation in transversely isotropic circular cylinders, part I: Theory, Part II: Numerical results, J. Acoust. Soc. Am., Vol. 37, pp. 1016-1026.

Moodie T. B. 1973. On the propagation, reflection and transmission of transient cylindrical shear waves in non-homogeneous fourparameter viscoelastic media, Bull. Austr. Math. Society, Vol. 8, pp. 397-411.

Mukhopadyaya, A.K., 1979. Note on the extensional vibrations of thing isotropic non- homogeneous circular plate with a circular role, Rev. Roumane, Sci. Tech. Ser. Mech. Appl., Vol.3. No. 24, pp. 507-513. 
Murayama S. and Shibata T. 1961. Rheological properties of clays, $5^{\text {th }}$ International Conference of Soil Mechanics and Foundation Engineering, Paris, France, 1, pp. 269 - 273.

Narian, S., 1980. A note on a torsion vibration of a non homogeneous isotropic cylinder, J. Sci. Res. B.H.U. (India), Vol .30, pp. 403-407.

Raous M., 1981. A periodic problem in viscoelasticity with variable coefficients, International journal of Engineering Science, Vol. 19, pp. 1145-1168.

Rao C. R. A. and Goda M. A. A., 1978. Generalization of Lamb’s problem to a class of inhomogeneous elastic half spaces, Proc. R. Soc. Lond., A 359, pp. 93-110.

Rostovtsev N.A. and Khranevskaia I. E., 1973. The plane problems for a non-homogeneous elastic circular domain, J. Appl. Math. Mech., Vol. 37, pp. 95-102.

Robert M. and Keller J. B., 1964. Asymptotic methods for partial differential equations; the reduced wave equation and Maxwell's equations, Report EM-194, Courant Institute of Mathematical Sciences, New York.

Singh B.M. and Dhaliwal R.S., 1980. Diffraction of anti plane sphere waves by a Griffith crack or a rigid strip lying at the interface of homogeneous and non-homogeneous elastic half spaces, Acta. Mech., Vol. 37. No. 4, pp. 137-144.

Singh G. and Singh A., 1979. Propagation, reflection and transmission of longitudinal waves in non-homogeneous five parameter viscoelastic rods, Indian Journal of Pure Applied mathematics, Vol. 11, pp 1249-1257.

Singh G., 1978. Some problems in wave propagation in non-homogeneous viscoelastic media, Ph. D. thesis, Punjabi University Patiala.

Schiffman R.L., Ladd C.C. and Chen A.T.F., 1964. The secondary consolidation of clay, Rheology and Soil Mechanics, Proceedings of the International Union of Theoretical and Applied Mechanics Symposium, Grenoble, Berlin, pp. 273 - 303.

Tsai Y.M. 1991. Longitudinal motion of a thick transversely isotropic hollow cylinder, Journal of Pressure Vessel Technology Vol. 113, pp.585-589.

Turban D. and Mengi V., 1977. The propagation of initially plane waves in non-homogeneous viscoelastic media, International Journal of Solid Structure, Vol. 13, No. 2, pp.22-30.

Victorov I. A., 1958. Rayleigh type wave on a cylindrical surface, Sov. Phys. Acoust., 4, pp. 131-136.

Watanabe K., 1981. Transient response of an inhomogeneous elastic halfspace, Bull. A.S.M.E. (Japan), Vol. 34, pp. 1537-1542.

White J.E. and Tongtaow C.1981. Cylindrical waves in transversely isotropic media, J. Acoustic Soc. Am. Vol.70. No. 4, pp. 11471155.

\section{Biographical notes}

Rajneesh Kakar received M. Phil. and Ph.D. from Punjab University Chandigarh, India in 1990 and 2013, respectively. He is Principal in the DIPS Polytechnic College, Tanda, Hoshiarpur (Punjab) India. He is having overall teaching experience of 23 years including professional colleges. His areas of interest include: Applied physics, applied mathematics, elasticity, viscoelasticity and fluid mechanics. He is a member of several learned societies. 